\title{
Design and Implementation of Data Analysis System Based on Mobile Internet
}

\author{
Yanling $\mathrm{Xu}$ \\ Department of Computer and Science, Yunyang Teachers' College, Shiyan 442000, China. \\ flyinggirl@sina.com
}

Keywords: Mobile Internet, Data analysis, MVC, Technical architecture, Database.

\begin{abstract}
This paper designed and partly implemented a mobile Internet data analysis system. The system includes user registration module, the user login module, the user password reset module, user management module, data receiving and statistics module, report generation and display module, real time acquisition news link module. This system refers to the classic MVC design pattern, using Struts2 as the controller to realize the persistent data using Hibernate technology, the realization of the data access technology is the way, the transaction management business logic components using the spring container, road. Through the framework of the SSH2 technology, the system not only has high degree of decoupling between the layers, but also has good maintainability and expansibility. In addition, the statistical report in the beauty of the front end of the plug-in was adopted in the system, the design method of using flexible and effective form of method Flexigrid plug-in; realize the asynchronous refresh the page using AJAX technology.
\end{abstract}

\section{Introduction}

At present, the entire society into the era of mobile Internet, it provides a new platform for communication and exchange for us. In today's increasingly competitive telecommunications industry, mobile Internet and telecom operators has brought new opportunities. [1] Telecom operators has its inherent advantages, namely, it is the most primitive data and user communication. As the network infrastructure of the owner, the premise of Internet data telecommunications operators can obtain user's, not revealing user privacy, performed the statistical analysis, I think, telecom operators will have their own user more deeply understand and master in the line of user behavior and network operating conditions. [2] Telecom operators can not only adjust or improve their quality of the network, but also can promote the personalized advertising customers, provide high quality service for the customer.

The vast amount of information carried over Mobile Internet and the high speed are providing unprecedented convenience for users, Mobile Internet is facing growing threat of lack of security. It is crucial to maintain and improve safety and security for Mobile Internet for it to thrive and develop. [3] At content level, users are facing increasing amount of malicious or spam content, jeopardizing public's interest in legitimate internet content. Therefore, Mobile Internet information security has become an important research topic. In this paper we first propose a framework for content security management system for Mobile Internet, and then discuss how to acquire relevant information from Mobile Internet in a fast and efficient manner, how to process and analyze the vast amount of information collected, how to quickly discover negative or illegal information within the network, and provide detection and early warnings for potential hot topics. [4] At the same time, we study how to perform audit and evaluation on the information content so that the relevant security management actions can be done.

This paper introduces the basic concept, and development status of the mobile Internet and SSH2 framework technology advantage, and then describes design of the main structure of mobile Internet data analysis system, and then introduces detailed design and code implementation of these modules, At the end, this paper describes how to design test cases to achieve comprehensive functional testing 
of the entire system and summarizes characteristics of the system and puts forward some aspects that need improvement of the system in the next step.

\section{Mobile world challenge}

Interaction Modes, Identity and Network environments are the main entities that the industry needs to create an environment for where they can interact seamlessly and invisibly from the end-user's point of view. This environment can be described as the Mobile World challenge, which is illustrated in the following Fig. 1.

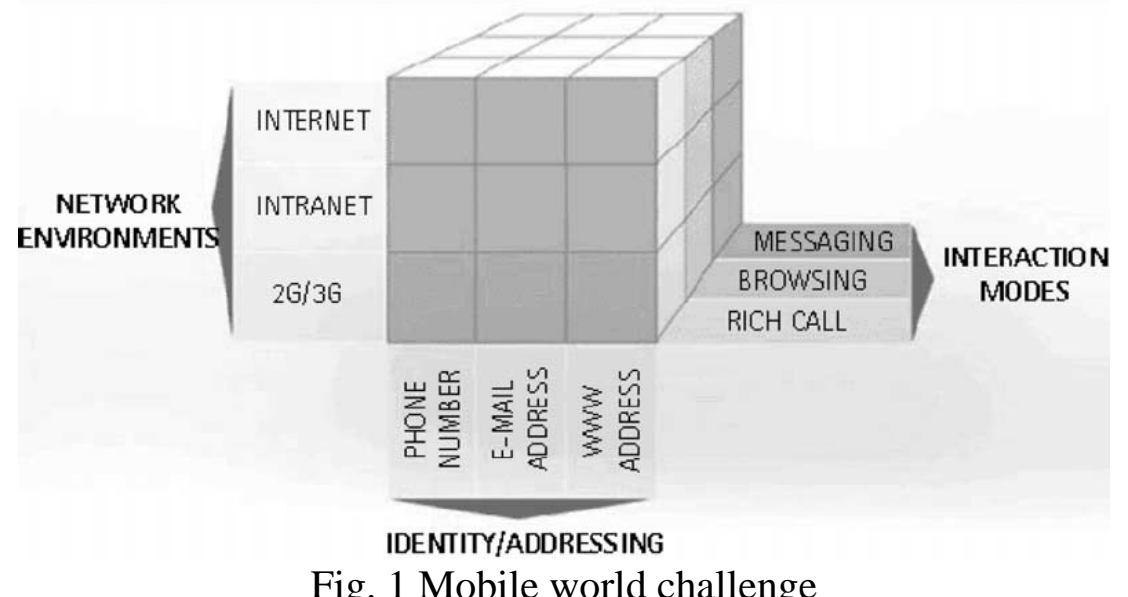

Fig. 1 Mobile world challenge

Also from MITA perspective this setting presents those areas that require close consideration in order to achieve a fully functional technical architecture for the Mobile World.

The primary objective is to provide user-friendly mobile Internet experience, when browsing messaging and that phone calls work seamlessly in any network environment and with any type of access. These essential aspects of user-friendly mobile entity are more closely identified in Fig. 2.

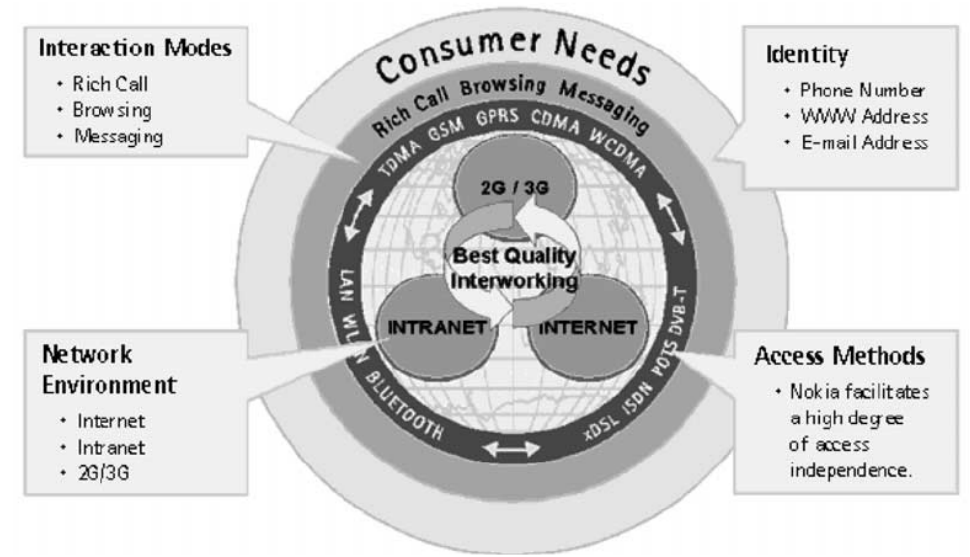

Fig. 2 The scope of Mobile Internet technical architecture

\section{Network environments}

The network environments: 2G/3G (mobile networks), Internet, and Intranet are the environment where the most of the user interaction will take place in the Mobile World. Furthermore, also these different environments need to work seamlessly together and enable evolution towards unified service networks. The interplay of networks, identity and interaction mode is modeled by the Mobile World challenge as illustrated in the figure above, which is a simple diagnostic framework for high-lighting the issues involved in interworking between layers (for example, in transferring a messaging session from one network environment to another). 


\section{The main interface of the system}

The system uses the Struts 1 framework, thus each module in the Model, Form and Service are inherits. When the user requests from the browser, Action received from the information on the page in the form of Form, then Form is converted to the corresponding Model. As shown in Fig. 3.

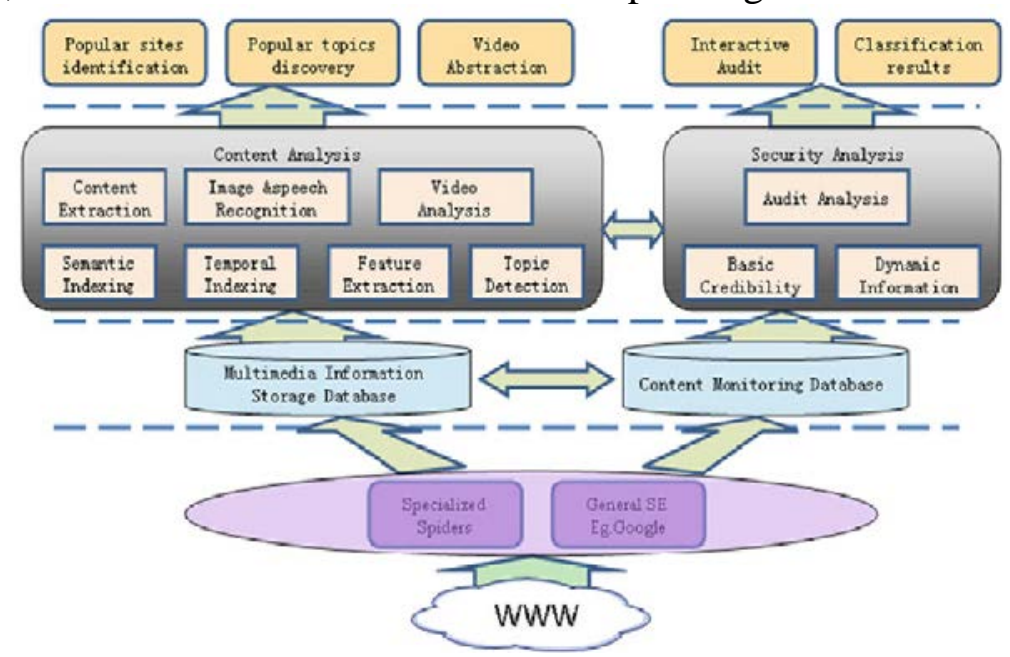

Fig. 3 Framework of content management system for Mobile Internet

\section{Analysis and processing subsystem}

Data content analysis and processing subsystem is primarily responsible and classification of language materials. We convert heterogeneous data content stored in the content database into homogenous data based on their metadata. The key issues are the definition and transformation of metadata. First we study the methods with XML based description language to describe the need of content management. On the other hand, since filters usually carry out their functions based on subjects, it is necessary to classify language materials by their subject. However, often a piece of language material may have unclear subject, or may be related to several subjects. Direct classifying attempts would not only fail to give out a clear category, but also shrink the coverage of the language material over possible subjects.

We can effectively solve this problem by the concept lattice's indirect description on language materials. From concept lattices we can extract assorted types of knowledge, such as implication rule, association rule, and classification rule etc. The concept lattice establishes the relationship between language materials and concepts, while the subjects are described by the concepts. Therefore, the concept lattice enables us to screen the language materials by assessing the relevance between the concept involved in the language materials and the information security. Language materials are to be selected based on the relationship between the screened subject and the concept in the lattice when to update and create the filters.

\section{Web page analysis technique}

The goal of webpage analysis is to extract useful information by removing control characters from the pages and identifying texts, images, audio and video contents for subsequent analysis, indexing and storage. Although the usual process is to decode HTML or XML files using existing technology, we will need to investigate ways to achieve high quality and high throughput analysis for particular types of web pages.

The main research focus of our system is the analysis web pages technique for podcasts and video sites for mobile phone. In order to effectively extract metadata information about texts, digits, images, and audio and video links, the system primarily uses intelligent analytical techniques for documents and the template interactive customization technique. For common unknown web sites, the system first decides major metadata information location within the site from typical content pages such as 
the HTML structure tags inside content table pages and the established DOM TREE. Next the system classifies useful metadata information using artificial intelligence algorithms based on page fide text and column content matching, in order to extract target information and establish page structures.

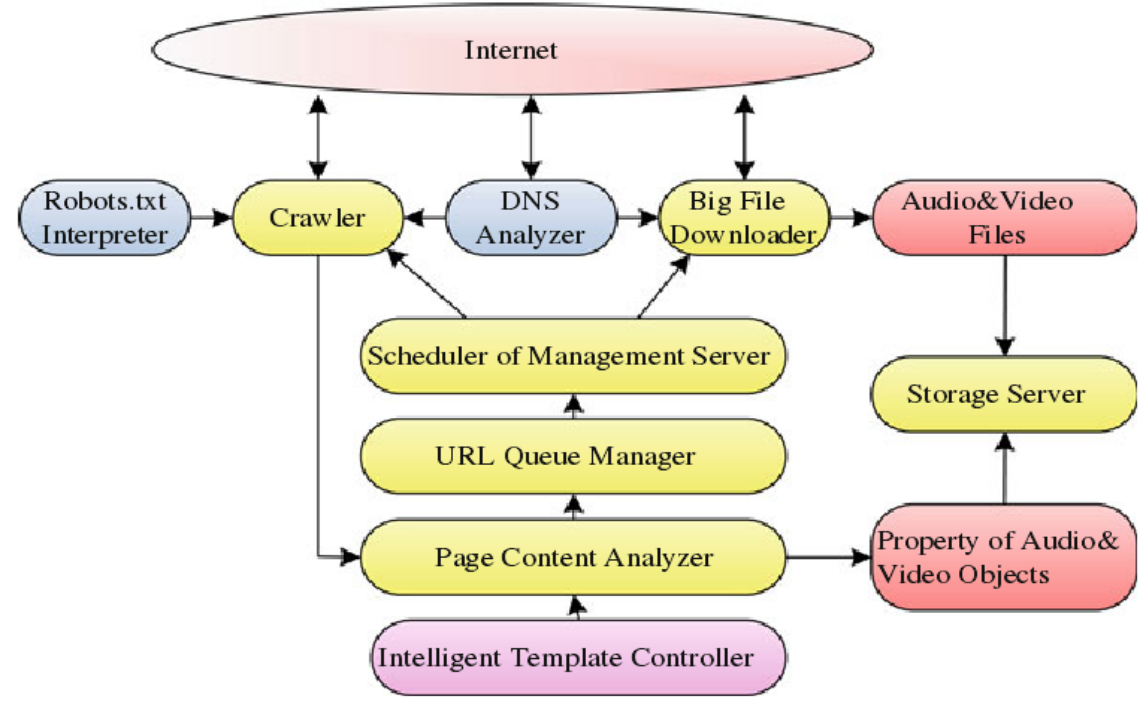

Fig. 4 Information crawling model for Mobile Internet

As shown in Fig. 4, the key to our system is to provide a probability model for data collected from social websites. Based on this probability model, we can adjust relevant parameters so that the model fits actual data as much as possible, thus it can be used to explain already collected data, and provide estimate for future data. This method can be used separately against hotness, spreading range, and comments of the information and establish different models; with the introduction of fame factor, the model can be used to describe temporal change of these statistics variables, in order to conduct comparison and selection for the information over the entire fame period. In the end, information such as hotness and spreading range is expressed as a probability, in order to describe users' attention to the information.

\section{Conclusions}

The usage of cellular phone has been expanded as wireless internet device recently. But its popular use is limited by some of reasons. The main reason is unshared data between data base servers. So, users can only access data that they know the link or location. In this paper, MRSS (Mobile Resource Sharing System) is presented to overcome the local limits of data and enhance data sharing methods. MRSS offers better service by using Context-Awareness Processor that transforms one instruction to many related instructions. MRSS is going to activate wireless internet service and provides a ubiquitous computing environment to customers using the mobile Internet.

\section{References}

[1] Katz, B., Lin, J. J.,\&Felshin, S. The START multimedia information system: Current technology and future directions. In Proceedings of the International Workshop on Multimedia Information Systems (MIS), 2002: 117-123.

[2] Lam, W., Meng, H., Wong, K.,\&Yen, J. (2001). Using contextual analysis for news event detetion. Interuatioual Journal on Intelligent Systems, 2001, 16(4): 525-546.

[3] Yang, J., Frangi, A\&Yang, J. (2005). KPCA plus LDA: A complete kernel Fisher discriminant framework for feature extraction and recognition. IEEE transactions on Pattern Analysis arid Machine Intelligence, 2005, 27(2): 230-244.

[4] Information on http://www.lemurproject.org. 